\title{
A Indicação Geográfica e o Paradoxo do Direito de Propriedade Intelectual no Estado do Pará: desafios e potencialidades do território paraense
}

\author{
Geographical Indication and the Paradox of Intellectual Property Right \\ in the State of Pará: challenges and potentials of the paraense territory
}

\author{
Manoel Enio Almeida Aguiar ${ }^{1}$ \\ Sidnei Cerqueira dos Santos ${ }^{1}$ \\ Franco Jefferds dos Santos Silva ${ }^{1}$ \\ Andrea Hentz de Mello ${ }^{1}$ \\ ${ }^{1}$ Universidade Federal do Sul e Sudeste do Pará, Marabá, PA, Brasil
}

\begin{abstract}
Resumo
A Propriedade Intelectual, no Brasil, é regulamentada pela Lei n. 9.279, de 14 de maio de 1996, conhecida como Lei da Propriedade Industrial (LPI). A mencionada lei regula direitos e obrigações relativos à Propriedade Industrial. É nesse ordenamento que existe a previsão da figura jurídica da Indicação Geográfica (IG). O Estado do Pará é uma unidade federativa extremamente rica em biodiversidade, produtos e serviços que poderiam ter sua origem protegidas pela IG, porém, possui, efetivamente, apenas um registro concedido. O objetivo deste trabalho é demonstrar o paradoxo existente entre a propriedade intelectual e as potencialidades existentes no Estado do Pará. Foram realizados estudos bibliográficos e exploratórios, cujos resultados demonstram que há muitas possibilidades no campo da IG. Conclui-se que esse paradoxo pode ser enfrentado a partir do avanço das articulações de diversos atores na busca pela institucionalização do direito de propriedade intelectual das riquezas distintivas paraenses.
\end{abstract}

Palavras-chave: Propriedade. Industrial. Biodiversidade.

\begin{abstract}
Intellectual property regulated in Brazil by Law n. 9,279 of May 14, 1996, known as the Industrial Property Law. The aforementioned law regulates industrial property rights and obligations. It is in this ordering that the legal figure of Geographical Indication (GI) exists. The State of Pará is a federative unit extremely rich in biodiversity, products and services that could have their origin protected by GI, but it has, in fact, only one granted registration. The aim of this paper was to try to demonstrate the paradox between intellectual property and potentialities in the State of Pará. Bibliographic and exploratory studies were conducted, the results of which show that there are many possibilities in the field of GI. It is concluded that this paradox can be faced from the advancement of the articulations of various actors in the search for the institutionalization of the intellectual property right of Pará distinctive riches.
\end{abstract}

Keywords: Industrial. Property. Biodiversity.

Área Tecnológica: Interdisciplinar. Propriedade Intelectual. Indicação Geográfica. 


\section{Introdução}

A Lei de Propriedade Industrial no Brasil não traz, de forma clara e direta, o conceito de Indicação Geográfica, mas a divide em duas espécies: Indicação de Procedência (IP) e Denominação de Origem (DO). A IP está relacionada com a reputação da região e a DO se relaciona com o meio geográfico, como os atributos do clima, solo, vegetação e um conjunto de know-how que são as técnicas tradicionais e constantes dos produtores ou prestadores de serviço de determinada região, que afetam o produto ou o serviço, conferindo-lhes características únicas e regionais. A IG, portanto, em sentido estrito, é uma espécie de Propriedade Industrial que, por sua vez, está compreendida nas definições de Propriedade Intelectual (BRASIL, 1996).

Na perspectiva das acepções de IG, Rocha Filho (2017) afirma que não há definições conclusivas na literatura mundial sobre o que é uma Indicação Geográfica, entretanto, Da Cruz (2017) define IG como um signo distintivo que tem a finalidade de proteger e de diferenciar produtos ou serviços, indicando sua área geográfica de extração, produção e/ou ocorrência, no caso dos serviços. Na perspectiva de Costa (2014), a Indicação Geográfica é um instrumento que apresenta potencial para proteger diversos aspectos de produtos e serviços, como a qualidade de produtos gerados em determinados territórios, incluindo o saber fazer e a forma de produção. De acordo com a Organização Mundial da Propriedade Intelectual (OMPI; INPI, 2016, p. 2), a Indicação Geográfica é definida como:

Indicações Geográficas, em seu conceito mais amplo, são indicações que identificam produtos ou serviços em razão de sua origem geográfica, e que incorporam atributos como reputação e fatores naturais e humanos, proporcionando produtos ou serviços com características próprias, que traduzem a identidade e a cultura de um espaço geográfico.

Ainda explorando os conceitos básicos do símbolo distintivo que trata esta pesquisa sob a ótica de diferentes autores, Locatelli (2009) define Indicações Geográficas como direitos relativos à propriedade industrial que atua como signos distintivos, ou seja, essa espécie de propriedade intelectual diferencia os produtos e os serviços por sua origem geográfica, afirmando ainda que a possibilidade do reconhecimento e da proteção jurídica da Indicação Geográfica constitui um importante instrumento de desenvolvimento econômico em diversos países em que a proteção é regulamentada. No sentido mais técnico de suas origens contemporâneas, o termo Indicação Geográfica, em seu conceito mais restrito, está relacionado à definição estabelecida no Acordo sobre Aspectos dos Direitos de Propriedade Intelectual Relacionados ao Comércio (em inglês Trade-Related Aspects of Intellectual Property Rights - TRIPS).

Embora o conceito de Indicação Geográfica seja distinto das definições de território, entende-se que não dá para dissociar o estudo de IG com a contextualização e pesquisa de territórios e desenvolvimento local sustentável. Dessa forma, Thaines (2013) afirmou que o reconhecimento de IG pode ser considerado uma ferramenta de fomento para o desenvolvimento regional, conforme apontam os conceitos de Fischer (2002). As IGs se revelam como fonte de desenvolvimento econômico, social, cultural e humano em determinados territórios, principalmente com a participação dos atores locais (DONA; ZAMBON, 2019; MARINS; CABRAL, 2015). 
A Organização Mundial da Propriedade Intelectual (OMPI, 2016), órgão ligado à Organização das Nações Unidas (ONU), é responsável pela proteção dos direitos de Propriedade Intelectual (PI) em nível internacional e funciona como uma agência que tem a tarefa de promover a atividade intelectual criativa e a transferência de tecnologia para os países em desenvolvimento, com o intuito de promover o desenvolvimento econômico, social e cultural. Diversos produtos podem ser protegidos por meio de Indicações Geográficas, como produtos industriais e agrícolas. Na legislação brasileira também podem ser protegidos artesanatos e serviços (BRASIL, 1996).

No Brasil, a Indicação Geográfica é considerada de natureza declaratória, isto é, ela é desenvolvida pelos produtores e prestadores de serviços e identificada pelos consumidores. O Instituto Nacional da Propriedade Industrial (INPI), órgão do Ministério da Economia, é responsável pelo registro das Indicações Geográficas concedidas em todo o território brasileiro, inclusive o registro de Indicação Geográfica de produtos estrangeiros (BRASIL, 1996).

O Estado do Pará está localizado na Região Amazônica e possui um grande potencial agroambiental e cultural. Os produtos e serviços são extremamente tradicionais, tanto na sua essência como no modo de serem produzidos, mas os produtores e prestadores de serviços, ainda que organizados em sistemas de produção, detêm pouco conhecimento sobre as possibilidades de proteção intelectual da origem das riquezas paraense. Nesse contexto, o objetivo deste trabalho é demonstrar o paradoxo existente entre a propriedade intelectual e as potencialidades existente no Estado do Pará.

\section{Metodologia}

Este estudo foi realizado por meio de uma abordagem descritiva qualitativa (CRESWELL, 2010), a partir de análise das principais referências disponíveis na literatura sobre o objeto pesquisado, incluindo obras publicadas em bancos de dados virtuais, bases de periódicos científicos, legislações e informações disponíveis nas plataformas do Instituto Nacional da Propriedade Industrial (INPI) e da Fundação Amazônia de Amparo a Estudos e Pesquisas (FAPESPA). A pesquisa foi realizada no período de maio de 2018 a agosto de 2019.

\section{Resultados e Discussão}

Após 23 anos da instituição da LPI, o Estado do Pará detém apenas um único registro concedido de Indicação Geográfica. Trata-se do registro de Indicação de Procedência do fruto cacau (Theobromo cacao L.) proveniente do município de Tomé-Açu, situado na mesorregião do nordeste paraense, cuja publicação oficial de concessão do registro ocorreu somente no ano de 2019 (INPI, 2018). 
Figura 1 - Cacau de Tomé-Açu recebe primeira IG do Pará

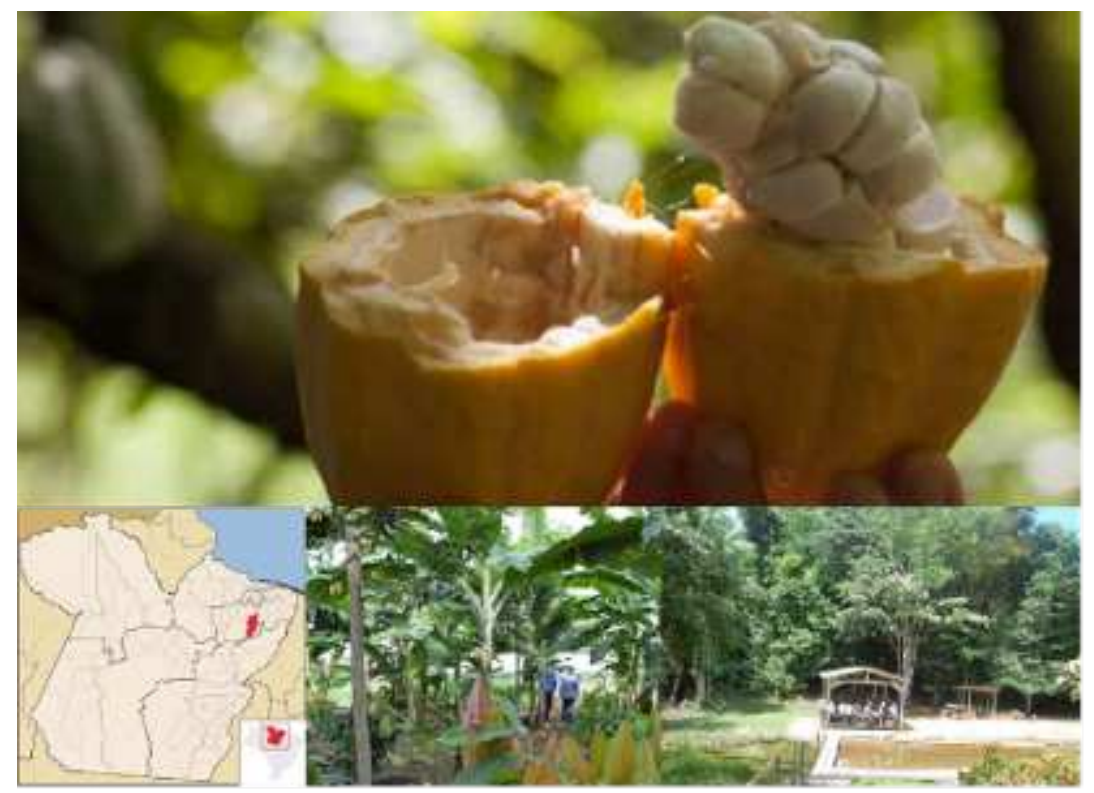

Fonte: Ideflor-Bio (2018)

Ainda em 2019 foram protocolados junto ao INPI duas novas solicitações de reconhecimento de Indicação Geográfica no âmbito do Estado do Pará: Queijo da região da Ilha do Marajó e Farinha do Município de Bragança. Ainda assim, o Estado do Pará está muito longe de ser considerado referência na proteção intelectual da origem de seus produtos e serviços como signos distintivos.

A Tabela 1 ilustra a Indicação Geográfica do cacau de Tomé-Açu e os dois produtos que tiveram os pedidos de registro protocolados junto ao INPI. A IG no território paraense representa pouco mais de $1 \%$ do total de IGs reconhecidas no Brasil (INPI, 2019), ou seja, existe um desafio muito grande a ser vencido no âmbito de novos reconhecimentos de IGs no Estado do Pará. Os estudos demonstram que não basta o reconhecimento por si só de uma IG.

Tabela 1 - Registros de Indicação Geográfica concedidos e solicitados

\begin{tabular}{cccc} 
Produto & MunicíPIO & ANo Da Solicitação & ANO Do RECONHECimento \\
Cacau & Tomé-Açu & 2014 & 2019 \\
Farinha & Bragança & 2019 & --- \\
Queijo & Ilha do Marajó & 2019 & --- \\
\hline
\end{tabular}

Fonte: INPI (2019)

No caso particular do Queijo de búfala da Ilha do Marajó e da Farinha de Bragança, por exemplo, produtos extremamente singulares e tradicionais, mesmo diante da perspectiva de obtenção do selo de IG pelos produtores, é possível inferir que há um longo caminho a ser percorrido para a consolidação das IGs como um instrumento de Propriedade Intelectual $e$ de desenvolvimento local (DA CRUZ et al., 2015), e essa etapa é denominada pós-certificado 
(AVENI, 2019). Entretanto, Da Cruz (2017, p. 25), em seus estudos sobre Indicações Geográficas na Amazônia, afirma que "[...] o uso das Indicações Geográficas como estratégia de desenvolvimento territorial é uma tendência mundial, tendo na União Europeia seu epicentro e se espraiando para várias regiões e países no mundo, a exemplo do Brasil".

A Figura 2 apresenta a dimensão do território paraense e a localização geográfica da IG do Cacau de Tomé-Açu (BR4020140000107), publicado na Revista da Propriedade Industrial em 2018, bem como das microrregiões referentes aos pedidos de IG da Farinha de Bragança e do Queijo de búfala da Ilha do Marajó. Percebe-se que as articulações dessas IGs têm ocorrido em regiões mais ao Norte e ao Nordeste do estado, regiões pioneiras no processo de colonização do Estado do Pará.

Figura 2 - Microrregiões paraenses e a localização das Indicações Geográficas registradas e em processo de registro no Estado do Pará
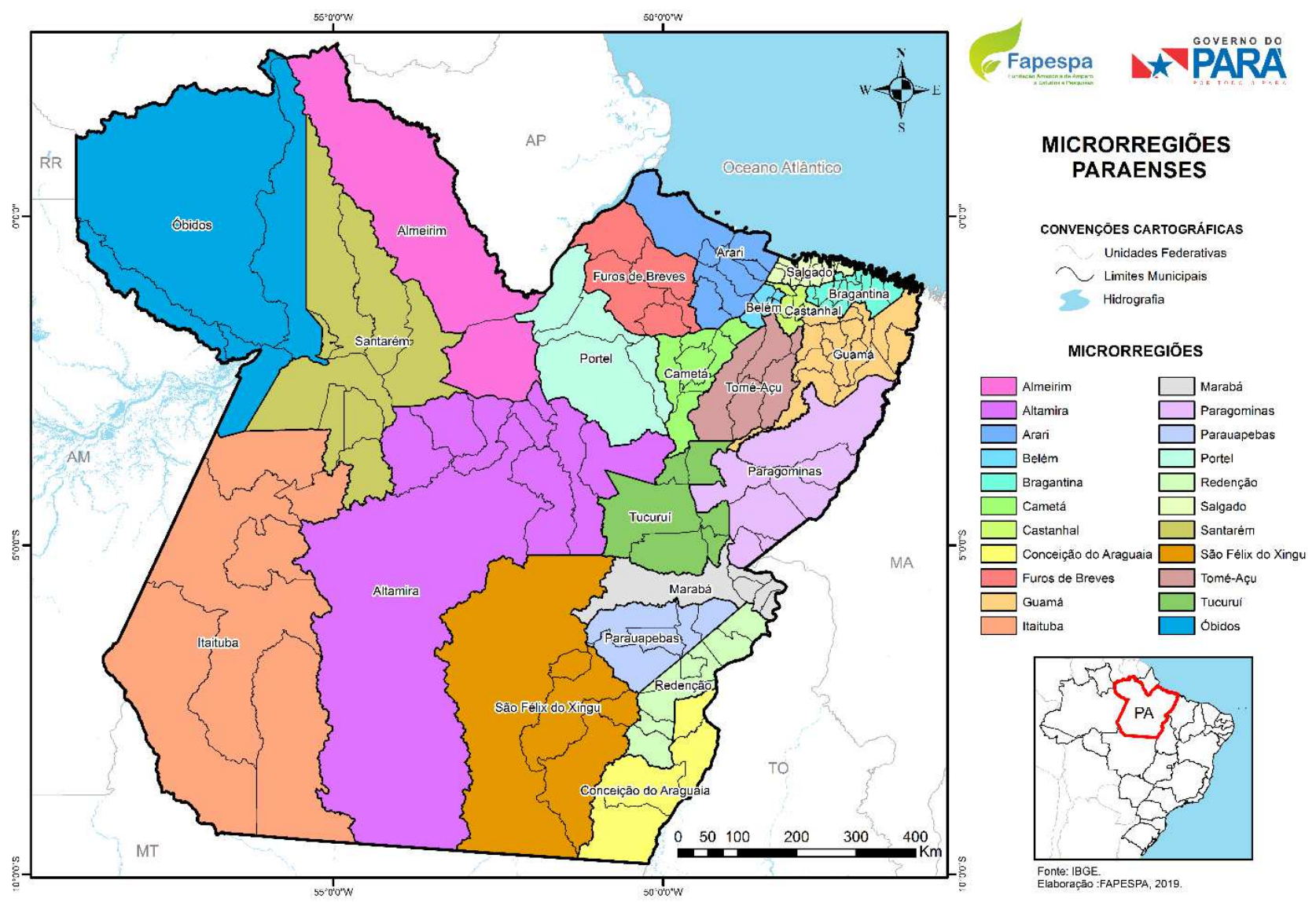

Fonte: Adaptada de FAPESPA (2019)

A OMPI e a INPI (2016) elencam uma série de possíveis repercussões positivas advindas do reconhecimento das Indicações Geográficas, como o aumento do valor agregado dos produtos/ serviços, a preservação das particularidades dos produtos/serviços, o patrimônio regional, o estímulo aos investimentos local, o aumento da autoestima da população local, a criação de vínculo de confiança com o consumidor, a melhoria no processo de comercialização dos produtos/serviços e uma maior competitividade no mercado nacional e internacional. 
Para Da Cruz (2017), obter o registro de Indicação Geográfica não é tão difícil, mas regulamentá-la e transformá-la em instrumento de desenvolvimento local é o grande desafio. Locatelli (2009), corroborando com os aportes de Da Cruz (2017), afirma que as Indicações Geográficas, por si só, não trarão o desenvolvimento e nem mudarão a conjuntura econômica e social, caracterizada pela pobreza e pela exclusão social, mas poderão agregar valor e promover o produto no mercado. Na mesma linha de entendimento, Ascensão (2009) afirma que essa espécie de propriedade industrial interessa de modo diferente aos países em desenvolvimento. $\mathrm{O}$ autor tenta evidenciar que a IG tem interesses muito desiguais para diferentes países.

Na tentativa de responder aos novos desafios da relação entre IG e desenvolvimento territorial, Cerdan (2013) cogita o desenvolvimento rural de forma mais holística, considerando-o como um duplo processo de integração econômica e especialização/especificação dos recursos do território. Nesse sentido, o autor demonstra a necessidade de análise e de avaliação da contribuição das estratégias de valorização dos produtos e serviços com forte identidade, ou seja, produto/serviço típico ou suscetível de Indicação Geográfica, em processo de desenvolvimento territorial (CERDAN, 2013).

Há algumas possibilidades legais que, se agregadas e desenvolvidas de forma colaborativa, a partir da efetivação de políticas públicas institucionais, podem potencializar os ganhos que as IGs tendem a proporcionar. Umas dessas possibilidades é o Decreto Federal n. 6.040, de 7 de fevereiro de 2007, que Institui a Política Nacional de Desenvolvimento Sustentável dos Povos e Comunidades Tradicionais (BRASIL, 2007). Nota-se que a Indicação Geográfica está na fase de "alvorecer", não apenas no Estado do Pará, mas também no Brasil, talvez vista como algo desconhecido por muitos segmentos sociais e até acadêmico, sobretudo na Região Amazônica, especialmente no Estado do Pará.

Da Cruz (2017) considera incipientes os conhecimentos, as iniciativas e as políticas direcionados às IGs no Estado do Pará. Nesse sentido, o autor teve a iniciativa de promover o primeiro Seminário de Identificação Geográfica do Estado do Pará, realizado no mês de maio de 2016, na cidade de Belém, com o propósito de traçar estratégias e ações para impulsionar a expansão desse signo distintivo. Na ótica do autor, o seminário possibilitou observar que há um movimento que tende a ganhar força, haja vista algumas ações que começam a ser elaboradas, como o Fórum Técnico de Indicação Geográfica e Marcas Coletivas do Estado do Pará1, ocorrido nos anos de 2018 e 2019 (DA CRUZ, 2017).

O Estado do Pará é rico em biodiversidade e oferece grandes oportunidades para o desenvolvimento de diferentes produtos, processos ou prestação de serviços, conforme se demonstra na Figura 3. Para Locatelli (2016), é imprescindível que haja mais divulgação desses signos geográficos para que o consumidor brasileiro, de um modo geral, comece a valorizar não somente esses produtos/serviços, mas também a cultura e a tradição que estão relacionadas a eles. Maiorki e Dallabrida (2015) também sustentam que as IGs deveriam ser mais divulgadas para impulsionar a busca por produtos e serviços originários do processo de desenvolvimento territorial.

\footnotetext{
${ }^{1}$ Fórum criado oficialmente por meio da Portaria n. 338/2016, publicada no Diário Oficial do Estado do Pará, em 7 de outubro de 2016, pela Secretaria de Estado de Turismo. A mencionada portaria define, entre outras coisas, a finalidade, a composição e a direção do Fórum (PARÁ, 2016, p. 46).
} 
Figura 3 - Uso e cobertura do solo paraense

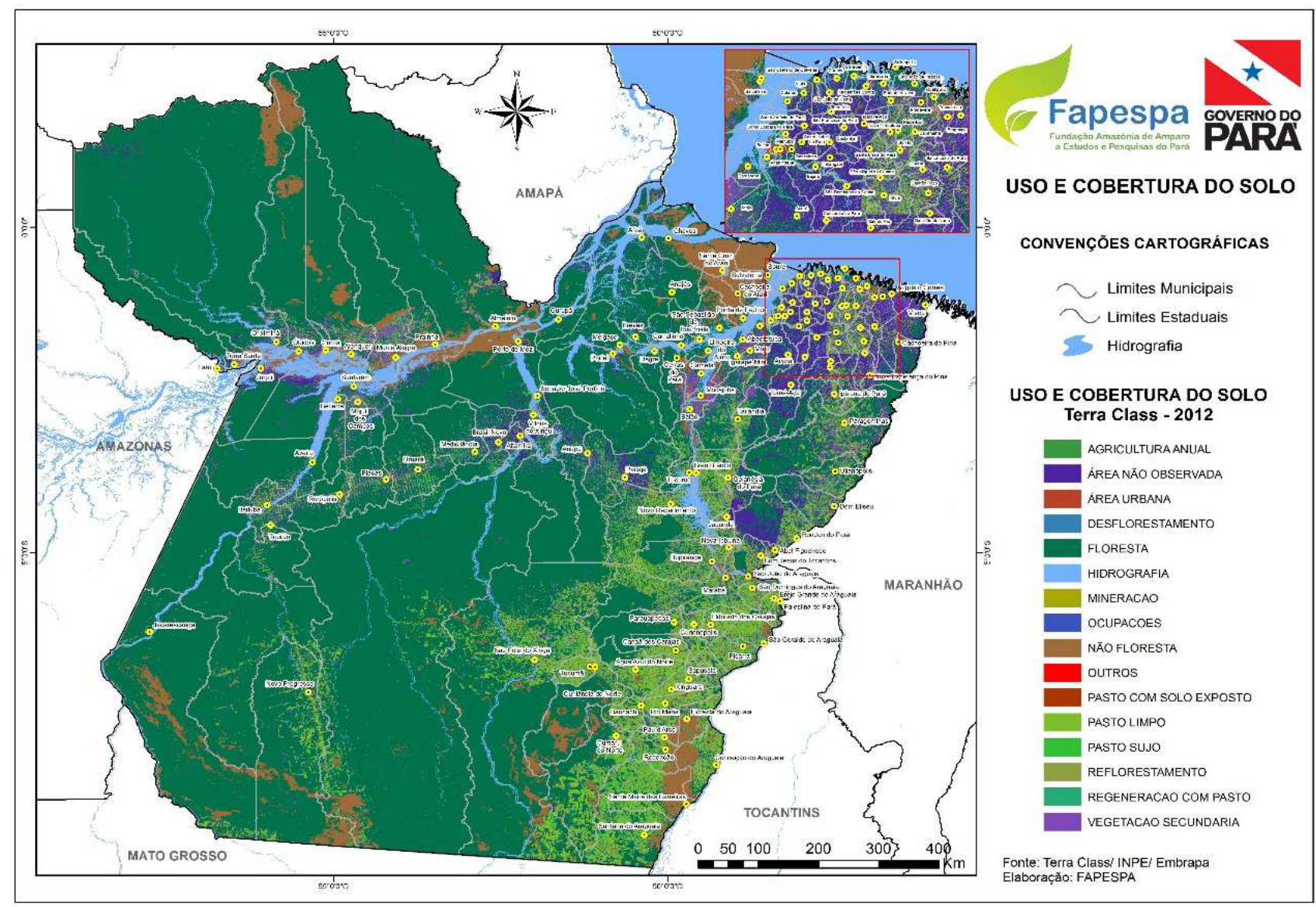

Fonte: FAPESPA (2019)

A disseminação das Indicações Geográficas no Estado do Pará, seja por meio de informações gerais ou por produção científica, pode ser o elo necessário ao fomento desse signo distintivo e da diminuição do atual quadro de déficit de proteção intelectual no território paraense. Nesse contexto, é fundamental a participação de produtores, instituições, governo, academia e demais segmentos, de forma integrada e coletiva, no estudo de produtos e serviços que apresentem potencial de proteção como IG no território paraense, visando a reduzir o atual quadro paradoxal: Estado rico e proteção intelectual incipiente. Além disso, é importante reforçar os aspectos positivos que o desenvolvimento de IG pode proporcionar, especialmente a partir de incentivos e políticas públicas associadas ao desenvolvimento regional.

A Tabela 2 apresenta alguns produtos com potencialidade de registro de Indicação Geográfica a partir da análise da biodiversidade paraense (FAPESPA, 2019). Ressalta-se que a lista disposta na Tabela 2 não é taxativa, tão pouco os produtos e serviços elencados são definitiva e indiscutivelmente suscetíveis à Indicação Geográfica, entretanto, são tradicionalmente conhecidos como produtos e serviços com uma importante carga de tipicidade regional. 
Tabela 2 - Produtos e serviços com potencial de Indicação Geográfica no Estado do Pará

\begin{tabular}{|c|c|}
\hline Produto/Serviço & ReGIÃo/MunicíPIO \\
\hline Abacaxi & Floresta do Araguaia \\
\hline Açaí das Ilhas & Região das Ilhas (também em outras regiões) \\
\hline Artesanatos Marajoara & Distrito de Icoaraci - Belém \\
\hline Bolinho de Piracuí de Prainha & Santarém \\
\hline Brinquedos de Miriti & Abaetetuba \\
\hline Carne Bovina & Xinguara (também em outras regiões) \\
\hline Coco Babaçu & São Domingos do Araguaia \\
\hline Cupuaçu & Tomé Açú (também em outras regiões) \\
\hline Minério de Cobre & Marabá \\
\hline Minério de Ferro & Parauapebas \\
\hline Pimenta do Reino & Tomé-Açu \\
\hline
\end{tabular}

Fonte: Elaborada pelos autores deste artigo (2019)

Para Pellin (2019), a prospecção de uma IG não se limita apenas a identificar os produtos em determinadas regiões, mas também identificar atores locais que podem se beneficiar direta ou indiretamente com o reconhecimento desse signo distintivo, como abordado nesta pesquisa. Conforme a própria legislação determina, uma série de estudos e normas deve ser desenvolvida para identificação e articulação do processo de IG. O estudo prospectivo pode possibilitar a antecipação de cenários estratégicos, escolha de oportunidades para estabelecer prioridade e avaliar lacunas a serem preenchidas, bem como os impactos da política tecnológica, como se inserem na sociedade, sendo capaz de influenciar de forma significativa nas atividades industriais, econômicas e na sociedade como um todo (QUINTELLA; GONÇALVES, 2011).

\section{Considerações Finais}

Em linhas gerais, o principal resultado observado é que os preceitos das Indicações Geográficas precisam ser melhor difundidos para reduzir o quadro paradoxal entre as possibilidades jurídicas da proteção intelectual dos símbolos distintivos do Estado do Pará e os produtos/serviços que efetivamente estão no foco de proteção pela LPI. A Pesquisa permitiu identificar que as IGs precisam ser fomentadas para comunidades locais dessa região, por meio da democratização do conhecimento técnico e da indução de novas possibilidades de Indicação Geográfica, especialmente nas Regiões Sul e Sudeste do Pará, que atualmente não detêm nenhum registro de IG, além de não haver registro de processo algum de articulação ou mobilização para tal. Este estudo identificou também que há um número muito reduzido de produção cientifica sobre IG na Região Amazônica, principalmente no Estado do Pará, o que reforça o paradoxo existente entre a IG e as potencialidades (produto/serviço) do Estado do Pará.

Refletir sobre o tema Indicação Geográfica e o paradoxo da Propriedade Intelectual no território paraense foi a mola propulsora deste trabalho, no intuito de despertar e de sensibilizar a sociedade organizada do Estado do Pará, entre eles: atores sociais e políticos interessa- 
dos em proteger o saber-fazer, os costumes, as tradições, as riquezas naturais e as distintivas. O fortalecimento de ações institucionais de proteção intelectual da origem de produtos e serviços distintivos é essencial para o desenvolvimento regional e para a afirmação de comunidades locais e tradicionais. É de grande relevância a difusão dessa possibilidade de proteção jurídica que a IG proporciona aos produtores e prestadores de serviço estabelecidos no local, por meio de trabalhos técnicos, científicos, da mídia de rádio e TV, em sites públicos, principalmente quando é complementada com políticas institucionais efetivas de geração de emprego e renda de forma sustentável e considerando a pluralidade de costumes e as tradições dos povos da Região Amazônica.

Novos estudos precisam ser realizados com intuito de analisar a consolidação da Indicação Geográfica do Cacau de Tomé-Açu e a potencialidade de novos produtos e serviços de IG na Região Amazônica, principalmente no Pará, usando outras ferramentas metodológicas (e.g. questionário, entrevista), com o intuito de promover o desenvolvimento de territórios e a proteção industrial de comunidades locais.

\section{Referências}

ASCENSÃO, J. O. Indicações geográficas e países em desenvolvimento In: DS - INSTITUTO DANNEMANN SIEMSEN DE ESTUDOS JURÍDICOS E TÉCNICOS. Propriedade Intelectual: plataforma para o desenvolvimento. Rio de Janeiro: Renovar, 2009. p. 101-121.

AVENI, A. Processo de pedido e projeto de pedido de indicações geográficas: menos burocracia e mais estratégia. Cadernos de Prospecção, Salvador, v. 12, n. 3, p. 695-705, 2019.

BRASIL. Código de Propriedade Industrial. Lei n. 9.279, de 14 de maio de 1996. Regula direitos e obrigações relativos à propriedade industrial. Disponível em: http://www.planalto.gov.br/ccivil_03/ leis/19279.htm. Acesso em: 21 maio 2019.

BRASIL. Decreto Presidencial n. 6.040, de 7 de fevereiro de 2007. Institui a Política Nacional de Desenvolvimento Sustentável dos Povos e Comunidades Tradicionais. Disponível em: http://www. planalto.gov.br/ccivil_03/_ato2007-2010/2007/decreto/d6040.htm. Acessa em: 21 maio 2019.

CERDAN, C. Indicações Geográficas e estratégias de desenvolvimento territorial. In: CERDAN, C. Indicações Geográficas: qualidade e origem nos mercados alimentares. Porto Alegre: EdUFRGS, 2013. p. 125-150.

COSTA, V. B. A indicação geográfica como ferramenta de valorização de território e proteção de comunidades locais com reputação de cultivo de plantas medicinais: o caso da região do Maciço da Pedra Branca no Estado do Rio de Janeiro. 2014. 114f. Dissertação (Mestrado Academia de Propriedade Intelectual, Inovação e Desenvolvimento) - Instituto Nacional da Propriedade Industrial, Rio de Janeiro, 2014.

CRESWELL, J. W. Projeto de Pesquisa: métodos qualitativo, quantitativo e misto. 3. ed. Porto Alegre: Artmed, 2010.

DA CRUZ, B. E. V. Alvorecer das indicações geográficas na Amazônia: a corrida pela IG do queijo artesanal do Marajó-PA. 2017. 388f. Tese (Doutorado em Geografia) - Universidade Estadual Paulista, São Paulo, 2017. 
DA CRUZ, B. E. V. et al. A identificação geográfica para o queijo do Marajó com estratégia de desenvolvimento territorial para a microrregião do Arari-Marajó, PA. Cadernos de Prospecção, Salvador, v. 8, n. 1, p. 158-168, 2015.

DONA, S. L.; ZAMBON, M. S. Um estudo sobre a indicação de procedência e a denominação de origem e sua influência sobre o desenvolvimento local. Teoria \& Prática: Revista de Humanidades, Ciências Sociais e Cultura, [s.l.], v. 1, n. 1, p. 1-18, 2019.

FAPESPA - FUNDAÇÃO AMAZÔNIA DE AMPARO A ESTUDOS E PESQUISAS. Anuário Estatístico do Estado do Pará. [2019]. Disponível em: http://www.fapespa.pa.gov.br/anuario_ estatistico/imagens/mapas/territorio/uso_e_cobertura_do_solo.jpg. Acesso em: 15 ago. 2019.

FISCHER, T. Gestão do desenvolvimento e poderes locais: marcos teóricos e avaliação. Salvador: Casa da Qualidade, 2002.

IDEFLOR-BIO. IDEFLOR-BIO capacita agricultores familiares de Tomé-Açu. 2018. Disponível em: https://ideflorbio.pa.gov.br/2018/03/ideflor-bio-capacita-agricultores-familiares-de-tome-acu/. Acesso em: 20 ago. 2019.

\section{INPI - INSTITUTO NACIONAL DA PROPRIEDADE INDUSTRIAL. Pedidos de Indicação}

Geográfica no Brasil. 2019. Disponível em: https:/www.gov.br/inpi/pt-br/servicos/indicacoesgeograficas/pedidos-de-indicacao-geografica-no-brasil. Acesso em: 15 ago. 2019.

INPI - INSTITUTO NACIONAL DA PROPRIEDADE INDUSTRIAL. Revista da Propriedade Industrial, Indicações Geográficas, n. 2.495, seção VI, p. 4-20, 2018. Disponível em: https:// www.gov.br/inpi/pt-br. Acesso em: 20 ago. 2019.

LOCATELLI, L. Indicações geográficas: a proteção jurídica sob a perspectiva do desenvolvimento econômico. Curitiba: Juruá, 2009.

LOCATELLI, L. O processo de consolidação das indicações geográficas no Brasil: lacunas e omissões da Lei n. 9.279/1996. Cadernos de Prospecção, Salvador, v. 9, n. 1, p. 152-158, 2016.

MAIORKI, G. J.; DALLABRIDA, V. R. A indicação geográfica de produtos: um estudo sobre sua contribuição econômica no desenvolvimento territorial. Interações, [s.l.], v. 16, n. 1, p. 13-25, 2015.

MARINS, M. F.; CABRAL, D. H. Q. O papel da Indicação Geográfica como propulsor da inovação e do desenvolvimento local: caso Vale dos Vinhedos. Cadernos de Prospecção, Salvador, v. 8, n. 2, p. 406-414, 2015.

OMPI - ORGANIZAÇÃO MUNDIAL DA PROPRIEDADE INTELECTUAL; INPI - INSTITUTO NACIONAL DA PROPRIEDADE INDUSTRIAL. Curso Geral de Propriedade Intelectual. DL 101P BR, módulo 5, Indicações Geográficas, 2016.

PARÁ. (Estado). Portaria n. 338/2016, publicada no Diário Oficial do Estado do Pará, em 7 de outubro de 2016, pela Secretaria de Estado de Turismo. [2016]. Disponível em: http://www. ioepa.com.br/pages/2016/10/07/2016.10.07.DOE_46.pdf. Acesso em: 17 ago. 2019.

PELLIN, V. Indicações Geográficas e desenvolvimento regional no Brasil: a atuação dos principais atores e suas metodologias de trabalho. Interações, [s.l.], v. 20, n. 1, p. 63-78, 2019.

QUINTELLA, C. M.; GONÇALVES, O. Processo microbiológico para captura de voláteis e produção de biopolímero proteico. BR PI011110000706. 2011. Disponível em: www.inpi.gov.br/. Acesso em: 16 ago. 2019. 
ROCHA FILHO, S. A. Indicações Geográficas: a proteção do patrimônio cultural na sua diversidade. São Paulo: Almedina, 2017.

SOUZA. V. M. Dinâmicas Territoriais e as quebradeiras de coco babaçu no Município de São Domingos do Araguaia-PA. 2014. 157 f. Dissertação (Mestrado em Dinâmicas Territoriais e Sociedade na Amazônia) - Universidade Federal do Sul e Sudeste do Pará. Disponível em: https:// pdtsa.unifesspa.edu.br/images/VALTEY.pdf. Acesso em: 21 maio 2019.

THAINES, A. H. Propriedade Intelectual: o desenvolvimento regional sob a óptica do reconhecimento da indicação geográfica e o case o Vale dos Vinhedos. Belo Horizonte: Arraes Editores, 2013.

\section{Sobre os Autores}

\section{Manoel Enio Almeida Aguiar}

E-mail:manoelenio@gmail.com Mestre em Propriedade Intelectual em 2020.

Endereço profissional: Avenida dos Ipês, s/n, Bairro Cidade Jardim, Marabá, PA. CEP: 68500-000.

\section{Sidnei Cerqueira dos Santos}

E-mail: sidnei.cerqueira@unifesspa.edu.br

Doutor em Biotecnologia em 2013.

Endereço profissional: Avenida dos Ipês, s/n, Bairro Cidade Jardim, Marabá, PA. CEP: 68500-000.

\section{Franco Jefferds dos Santos Silva}

E-mail: franco.silva@unifesspa.edu.br

Doutor em Engenharia Aeronáutica e Mecânica em 2011.

Endereço profissional: Folha 31, Quadra 7, Lote Especial, Nova Marabá, Marabá, PA. CEP: 68507-590.

\section{Andrea Hentz de Mello}

E-mail: andreahentz@unifesspa.edu.br Doutora em Ciências do Solo em 2006.

Endereço profissional: Avenida dos Ipês, s/n, Bairro Cidade Jardim, Marabá, PA. CEP: 68500-000. 